\title{
A Study on Post-Operative Wound Infections
}

\author{
Sagila Savithri Gangadharan*
}

Division of Microbiology, Regional Cancer Centre, Thiruvananthapuram, 695 011, India

*Corresponding author

\begin{tabular}{|l|}
\hline Ke y w o r d s \\
Etiology, SSI, \\
ESBL, Drug \\
resistance
\end{tabular}

\section{A B S T R A C T}

Sepsis in modern surgery continues to be a significant problem for healthcare practitioners across the globe. ${ }^{1}$ Since initial antimicrobial therapy usually remains empiric; the knowledge of prevailing susceptibility patterns at individual institutions is a must. To determine the etiology of surgical site infections (SSI) in patients admitted to a tertiary care teaching hospital, study its antibiogram and the prevalence of ESBL. 200 patients who developed SSI in a teaching hospital during a period of one year were prospectively studied. Clinical records were also reviewed to assess the surgical wound, associated risk factors and outcome. 200 patients, 88 males and 112 females, who developed SSI, were studied. Of the wounds studied, 103 were from clean- contaminated wounds. The most common pathogen was Staphylococcus aureus $(41.71 \%)$, followed by Klebsiella pneumoniae (19.43\%) and E. coli (17.14\%). MRSA prevalence was $28.77 \%$. ESBL prevalence was also high. Most gram negative bacilli other than Pseudomonas aeruginosa were found to be resistant to $1^{\text {st }}$ and $2^{\text {nd }}$ line drugs and sensitive mainly to carbapenems and

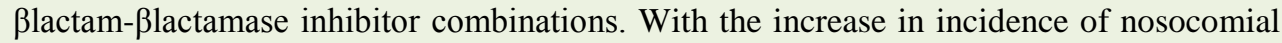
infections and multi drug resistance, a meticulous and periodic surveillance of SSI is called for.

\section{Introduction}

Infection has always been a feature of human life and sepsis in modern surgery continues to be a significant problem for healthcare practitioners across the globe (Lilani et al., 2005). The incidence of infection varies. Surgical site infectionis defined as infection occurring up to 30 days after surgery (or up to one year after surgery in patients receiving implants) (Alicia et al., 1999). Surgical site infections are a major part of Health Care Associated Infections (HCAI), accounting for approximately a quarter of all nosocomial infections, (Alicia et al., 1999; Ronald Lee Nichols, 2001; Suchitra Joyce et al., 2009) and also the third most common of nosocomial infections (Alicia et al., 1999; Suchitra Joyce et al., 2009). Post-operative wound infection delays recovery often increases length of stay (doubled) and cost (Singh et al., 2002) may produce lasting sequelae and require extra resources for investigations and management. Most of these infections are superficial and readily treated with a regimen of local care and antibiotics. However, soft tissue infections involving deeper layers like fascia and muscle can rapidly progress to systemic sepsis and prove fatal (Martone et al., 2001; Mohanty et 
al., 2004). These are the type of infections that require a wise choice of antimicrobials in addition to aggressive surgical debridement to limit tissue loss and preserve life (Martone et al., 2001; Mohanty et al., 2004).

Multi drug resistance is a dreaded problem in nosocomial infections including surgical site infections. Since initial antimicrobial therapy usually remains empiric, it is important to know, the prevailing susceptibility patterns of bacterial isolates at individual institutions by routine surveillance.

Intensive medical therapies and frequent use of antimicrobial drugs result in selection of resistant microbial flora. (Cruse, 1992; Cruse et al., 1980) Hospitals are reservoirs for strains of bacteria that are multi drug resistant. SSI due to resistant organisms has been a problem with an increase in the incidence of methicillin-resistant Staphylococcus aureus (MRSA), vancomycin - resistant Enterococcus (VRE) and Pseudomonas aeruginosa. (Agarwal, 1972; Cruse, 1992; Rao et al., 1975) There are however effective interventions for reducing the occurrence of HCAI.

Now with the use of antibiotics, a new era in the management of wound infections commenced. The search for new and more effective drugs continues even today. The pace, however, has slowed remarkably. Also, unfortunately, eradication of the infective plague affecting surgical wounds has not ended because of the insurgence of antibioticresistant bacterial strains and the nature of more adventurous surgical intervention in immune compromised patients and in implant surgery. Companies involved in the search for new antibiotics are also finding it increasingly difficult to keep up with the pace at which bacterial resistance renders their findings useless.

With the increase in incidence of nosocomial infections and multi drug resistance, a meticulous and periodic surveillance of various hospital acquired infections is called for.

\section{Aim of the study}

To determine the bacterial etiology of surgical site infections in a group of patients with surgical site infections admitted to Surgery and Obstetrics and Gynaecology (O\&G) in Medical College, Thiruvananthapuram and to study its antimicrobial susceptibility profile.

To find out the prevalence of ESBL among the organisms isolated.

\section{Materials and Methods}

\section{Study setting and design}

The prospective study was conducted on a group of inpatients from a tertiary care teaching hospital, in south Kerala after obtaining ethical clearance. Patients developing post-operative wound infections after a surgery done in the same hospital during a one year period 2008 -2009was included in the study. Only wounds which can be classified as clean, clean-contaminated or contaminated were included in the study. The microbiological methods used for culture, identification of the pathogen, finding the antimicrobial susceptibility profile and the methods for ESBL detection was also reviewed.

\section{Data collection}

Data regarding the demographic characteristics, type of surgery performed whether planned or emergency, classification of the surgical wound, other associated risk factors, antibiotic therapy given and outcome were collected from case records. Surgical wounds are classified as clean, clean 
contaminated, contaminated and dirty wounds

Clean: An uninfected operative wound in which no inflammation is encountered and the respiratory, alimentary, genital, or uninfected urinary tracts are not entered.

Clean-Contaminated: Operative wounds in which the respiratory, alimentary, genital, or urinary tracts are entered under controlled conditions and without unusual contamination

Contaminated: Open, fresh, accidental wounds including operations with major breaks in sterile technique

Dirty or Infected: Includes old traumatic wounds with retained devitalized tissue and those that involve existing clinical infection or perforated viscera

\section{Microbiological methods}

The present study was conducted in the Dept. of Microbiology, Government Medical College, Thiruvananthapuram. 200 operated cases in Surgery and Obstetrics and Gynaecology were included in this study.

Two sterile swabs or in some cases, syringe aspirate were obtained from the post-operative infected wounds after informed written consent of the patient and transported to the lab and processed without delay.

Samples were subjected to gram staining and culture. For culture, the specimen was inoculated into the following media-

5\% Blood agar (BA)

Macconkeyagar (MA)

Salt agar (SA)

Chocolate agar (CA)

Glucose broth (GB)

Robertson Cooked meat medium (RCM)

After overnight incubation, the plates were read for growth. Smears were made from liquid media if it appeared turbid and subculture done accordingly. The colonies were then identified by the conventional microbiological methods. Antimicrobial susceptibility testing was done by KirbyBauer disc diffusion method and interpretation was done according to CLSI guidelines.

ESBL detection by Double Disc Synergy Test (DDST): Synergy was determined between a disc of amoxicillin-clavulanate $(20 \mu \mathrm{g} / 10 \mu \mathrm{g})$ (augmentin) and a 30- $\mu$ g disc of three thirdgeneration cephalosporin antibiotics namely Ceftriaxone, Cefotaxime and Ceftazidime placed at a distance of $20 \mathrm{~mm}$ from center to center on a Mueller-Hinton Agar (MHA) plate swabbed with the test isolate. Clear extension of the edge of the inhibition zone of cephalosporin toward the augmentin disc resulting in a characteristically shaped zone referred to as 'keyhole' was interpreted as positive for ESBL production.

Control strains of Staphylococcus aureus Atcc 25923, E coli ATCC 25922, Pseudomonas aeruginosa ATCC 27853 and ESBLproducing Klebsiella pneumoniae ATCC 700603 were used throughout the study.

\section{Results and Discussion}

\section{Patients' characteristics}

As per the inclusion criteria, pus samples were taken from 200 patients who had Surgical Site Infections (SSI). Of this, 127 cases were taken from Surgery and 73 cases from Obstetrics and Gynaec (O\&G). Age of the patients ranged between 20-89years with a median age of patients developing SSI in Surgery cases being 45.0 and in O\&Gbeing 29.0. 88 patients were males and 112 patients were females with a male to female ratio1: 1.3.The age distribution of patients developing SSI is given in table 1. Most of the SSI studied was 
from clean contaminated wounds. The tabulation of SSI based on the wound class is given in table 2 .

Association of certain risk factors like diabetes mellitus, previous hospitalization, extremes of age and emergency surgery was studied. It was seen that 82 patients $(41 \%)$ had unplanned emergency surgery, 75 patients (37.5\%) had previous history of hospitalization and 74 patients (37\%) had diabetes mellitus. The categorization of these risk factors is given in table 3 .

\section{Etiology}

Bacterial pathogens were isolated from 147 individuals. A single etiologic agent was identified in $120(60 \%)$ patients, multiple agents were found in $27(13.5 \%)$, and culture negative in 53(26.5\%). A high preponderance of aerobic bacteria was observed. Among the common pathogens were Staphylococcus aureus (73 patients, 41.71\%), Klebsiella pneumoniae (34 patients, 19.43\%) and E. coli (30 patients, 17.14\%). The detailed etiology is given in table 4 .

\section{Antimicrobial resistance}

The antibiotic resistance pattern of gram positive cocci and gram negative bacilli to the commonly tested antibiotics is given in tables $5 \mathrm{a}$ and $5 \mathrm{~b}$. Because of the increased resistance pattern to III generation Cephalosporins, Class I ESBL was tested for the $E$ coli and $K$ pneumoniae isolates using DDST. 18 (60\%) E coli and 22 (64.71\%) Klebsiellapneumoniae were found to be ESBL producers by this method.

\section{Outcome}

191(95.5\%) patients had uneventful recovery following appropriate antibiotic therapy, while $9(4.5 \%)$ patients died due to fulminant sepsis before the organism was isolated.

This study provides data on the microbiology and antibiotic susceptibility pattern in a group of patients who developed SSI in the institution. The development of postoperative SSI is known to be multifactorial, arising from a complex relationship and delicate interplay between host, microbe and environmental factors. SSIs may occur following any surgical incision, even after the use of minimally invasive techniques, so SSIs need to be reported through systematic monitoring. The dominant causative microorganisms and treatment options have changed over time. Today, most common pathogens are resistant to common antibiotics and there is a need for a high index of suspicion, prompt operative intervention, appropriate antibiotic treatment and proper resuscitation. (Josep Ballus et al., 2015)

During the course of one year, a total of 200 surgical site infections (SSI) were studied. Samples were collected only from those patients with confirmation of SSI by the surgeon.Gram negative bacilli predominated in Surgery accounting for $53 \%$ of isolates. This is in accordance with previous studies showing that gram negative bacilli predominate in abdominal surgeries. (Josep Ballus et al., 2015) In O\&G, gram positive bacteria predominated probably because most of the surgeries were clean surgeries. (Nichols, 1984) A number of studies in literature indicates gradual increase in the emergence of antibiotic resistant microorganisms in surgical patients. (Agarwal, 1972; Rao et al., 1975)

Most of the isolates obtained in Surgery were resistant to multiple drugs. This could be due to the higher antibiotics given to patients on admission due to complications, or due to gastro- intestinal surgeries performed (Josep Ballus et al., 2015). 
The following antibiotic discs were used

\section{- Penicillin (10IU)}

- Ampicillin (10 $\mu \mathrm{g})$

- Erythromycin $(15 \mu \mathrm{g})$

- Gentamicin (10 $\mu \mathrm{g})$

- I $^{\text {st }}$ genCephalosporins $(30 \mu \mathrm{g})$

- Cotrimoxazole $(1.25 / 23.75 \mu \mathrm{g})$

- Amikacin $(30 \mu \mathrm{g})$

- Ciprofloxacin $(5 \mu \mathrm{g})$

- Piperacillin-Tazobactam $(\mathbf{1 0 0 + 1 0 \mu g )}$
- III $^{\text {rd }}$ gen Cephalosporins(30 $\left.\mu \mathrm{g}\right)$

- Cefoxitin $(30 \mu \mathrm{g})$

- Vancomycin $(30 \mu \mathrm{g})$

- Rifampicin $(5 \mu \mathrm{g})$

- Clindamycin $(2 \mu \mathrm{g})$

- Linezolid $(30 \mu \mathrm{g})$

- Cefoperazone-Sulbactum $(75+30 \mu \mathrm{g})$

- Imipenem $(10 \mu \mathrm{g})$

Table.1 Age wise distribution of patients

\begin{tabular}{|l|l|l|l|}
\hline Age distribution & Surgery & O\&G & Total \\
\hline $20-29$ & 22 & 40 & 62 \\
\hline $30-39$ & 23 & 17 & 40 \\
\hline $40-49$ & 34 & 9 & 43 \\
\hline $50-59$ & 25 & 3 & 28 \\
\hline $60-69$ & 17 & 4 & 21 \\
\hline $70-79$ & 4 & - & 4 \\
\hline $80-89$ & 2 & - & 2 \\
\hline Total & 127 & 73 & 200 \\
\hline
\end{tabular}

Table.2 Distribution of SSI based on wound

\begin{tabular}{|l|l|l|l|}
\hline Wound class & Surgery & O\&G & Total \\
\hline Clean & 71 & - & 71 \\
\hline Clean-contaminated & 33 & 73 & 106 \\
\hline Contaminated & 23 & - & 23 \\
\hline Total & 127 & 73 & 200 \\
\hline
\end{tabular}

Table.3 Categorization of patient associated risk factors

\begin{tabular}{|l|l|l|}
\hline & $\begin{array}{l}\text { No } \\
\text { patients }\end{array}$ & of $\%$ of patients \\
\hline Emergency surgery & 82 & $41 \%$ \\
\hline Previous h/o hospitalization & 75 & $37.5 \%$ \\
\hline Diabetes mellitus & 74 & $37 \%$ \\
\hline No risk & 32 & $16 \%$ \\
\hline Age $>70$ years & 6 & $3 \%$ \\
\hline
\end{tabular}


Table.4 Etiology

\begin{tabular}{|l|l|}
\hline Organism & Surgery \\
\hline Staphylococcus aureus & $\mathbf{7 3 ( 4 1 . 7 1 \% )}$ \\
\hline Staphylococcus epidermidis & $\mathbf{1 0}(\mathbf{5 . 7 1 \%})$ \\
\hline Enterococcus faecalis & $\mathbf{1 0}(\mathbf{5 . 7 1 \% )}$ \\
\hline Streptococci & $\mathbf{2 ( 1 . 1 4 \% )}$ \\
\hline Klebsiellapneumoniae & $\mathbf{3 4 ( 1 9 . 4 3 \% )}$ \\
\hline E coli & $30(\mathbf{1 7 . 1 4 \% )}$ \\
\hline Pseudomonas aeruginosa & $10(5.71 \%)$ \\
\hline Acinetobacterbaumannii & $6(3.43 \%)$ \\
\hline Total & $\mathbf{1 7 5}$ \\
\hline
\end{tabular}

Table.5a Antimicrobial resistance pattern of gram positive bacteria isolated from surgical wounds

\begin{tabular}{|r|l|l|l|l|}
\hline S. No & Antibiotic & $\begin{array}{l}\text { Staphylococcus } \\
\text { aureus }\end{array}$ & $\begin{array}{l}\text { Coagulase negative } \\
\text { Staphylococci }\end{array}$ & Enterococci \\
\hline 1. & Penicillin & $70(95.89 \%)$ & $9(90 \%)$ & $10(100 \%)$ \\
\hline 2. & Ampicillin & NT & NT & $10(100 \%)$ \\
\hline 3. & Gentamicin & $33(45.21 \%)$ & $8(80 \%)$ & $9(90 \%)$ \\
\hline 4. & Erythromicin & $43(58.90 \%)$ & $7(70 \%)$ & $10(100 \%)$ \\
\hline 5. & Oxacillin & $21(28.77 \%)$ & $6(60 \%)$ & NT \\
\hline 6. & Amikacin & $10(13.70 \%)$ & $2(20 \%)$ & NT \\
\hline 7. & Vancomycin & $0(0 \%)$ & $0(0 \%)$ & $0(0 \%)$ \\
\hline 8. & Linezolid & $0(0 \%)$ & $0(0 \%)$ & $0(0 \%)$ \\
\hline
\end{tabular}

Table.5b Antimicrobial resistance pattern of gram negative bacteria isolated from surgical wounds

\begin{tabular}{|c|c|c|c|c|}
\hline S. No & Antibiotic & Enterobacteriaceae & $\begin{array}{l}\text { Pseudomonas } \\
\text { aeruginosa }\end{array}$ & Acinetobacterbaumanii \\
\hline 1. & Ampicillin & $62(96.88 \%)$ & NT & $6(100 \%)$ \\
\hline 2. & Gentamicin & $43(67.19 \%)$ & $5(50 \%)$ & $5(83.33 \%)$ \\
\hline 3. & $\begin{array}{l}\text { Ist generation } \\
\text { Cephalosporins }\end{array}$ & $59(92.19 \%)$ & NT & $6(100 \%)$ \\
\hline 4. & $\begin{array}{l}\text { IIIrd generation } \\
\text { Cepahalosporins }\end{array}$ & $55(85.94 \%)$ & $\begin{array}{l}3(30 \%) \\
\text { (Ceftazidime) }\end{array}$ & $6(100 \%)$ \\
\hline 1. & Amikacin & $13(20.31 \%)$ & $2(20 \%)$ & $3(50 \%)$ \\
\hline 2. & Ciprofloxacin & $53(82.81 \%)$ & $4(40 \%)$ & $5(83.33 \%)$ \\
\hline 3. & Cotrimoxazole & $46(71.88 \%)$ & NT & $5(83.33 \%)$ \\
\hline 4. & Piperacillin/Tazobactam & $24(37.50 \%)$ & $2(20 \%)$ & $4(66.67 \%)$ \\
\hline 5. & Cefaperazone/Sulbactam & $16(25 \%)$ & $2(20 \%)$ & $2(33.33 \%)$ \\
\hline 6. & Imipenem & $0(0 \%)$ & $0(0 \%)$ & $1(16.67 \%)$ \\
\hline 7. & Meropenem & $0(0 \%)$ & $0(0 \%)$ & $1(16.67 \%)$ \\
\hline
\end{tabular}


When these patients developed infection, the pathogens were found to be multi drug resistant due to antibiotic selection.

$21(28.77 \%)$ of Staphylococcus aureus were methicillin resistant and $6(60 \%)$ of coagulase negative Staphylococci were methicillin resistant showing the rising prevalence of methicillin resistance among Staphylococci (Cruse et al., 1980; Mohanty et al., 2004; Lilani et al., 2005). But Vancomycin resistance was not encountered in the study.

As in other studies, (Cruse et al., 1980; Josep Ballus et al., 2015; Mandakini Pawar et al., 2008; Nichols, 1984) most of the Enterobacteriaceae isolates and Acinetobacterbaumanii were multi drug resistant and only sensitive to higher antibiotics like Piperacillin Tazobactum (Resistance- 24(37.50\%) among Enterobacteriaceae and 4(66.67\%) among Acinetobacter baumanii) and Cefaperazone Sulbactum (Resistance- 16(25\%) among Enterobacteriaceae and 2(33.33\%) among Acinetobacter baumanii). No carbapenem resistance was noted among Enterobacteriaceae and Pseudomonas aeruginosa but 1(16.67\%) Acinetobacter baumanii was found to be carbapenem resistant. Also gram negative bacilli demonstrated high prevalence of ESBL $(68.23 \%)$.

Death rate in this study was $4.5 \%$ which is slightly more than the WHO statistics $(2.1 \%)$, but comparable with Indian scenario. With resistant strains the mortality also increases as seen from studies conducted in India, $13.9 \%$ vs $1.8 \%$ seen with sensitive strains. (Mandakini Pawar et al., 2008)

From the tabulated risk factors, it was noted that diabetes mellitus was associated with SSI in $74(37 \%)$ and correlates with other studies (Alicia J. Mangram et al., 1999; Suchitra
Joyce et al., 2009), prior hospitalization in 75 (37.5\%) (Alicia J. Mangram et al., 1999), emergency surgery in $82(41 \%)$. (Alicia J. Mangram et al., 1999) Other risk factors like immunosuppression, steroid therapy, malnutrition and anemia were seen in only a minority. The study gives us insight into the bacterial flora isolated and their resistance pattern in postoperative patients in this institution. This study can be further extended to other elective and emergency surgical procedures for a considerable duration. Surveillance of SSI with feedback of appropriate data to surgeons would be desirable to reduce the SSI rate.

This study has certain limitations. The most important is that we used for microbiological sampling, double skin swabs in most cases instead of tissue or aspiration sampling of infected tissue. Also follow up cultures and molecular typing of isolates was not done.

\section{Acknowledgement}

The author acknowledges the members of the Department of Microbiology, Surgery and Gynaecology of Medical College, Hospital, Thiruvananthapuram for their contribution to the study.

With the increase in incidence of nosocomial infections and multi drug resistance, a meticulous and periodic surveillance of various hospital acquired infections is called for. Periodic surveillance of SSI will guide the Infection Control Committee in laying down strict guidelines to further decrease the SSI in hospitals, which is an indicator of health care.

\section{References}

Agarwal SL. Study of postoperative wound infection. Indian J. Surg. 1972; 34: 31420. 
Alicia J. Mangram et al., Guideline for prevention of surgical site infection 1999. Infect Control Hosp Epidemiol. 1999; 20:247-80. Available online at www.cdc.gov/ncidod/hip.

Cruse PJE 1992. Classification of operations and audit of infection. In: Taylor EW, editor. Infection in Surgical Practice. Oxford: Oxford University Press, pp. 17.

Cruse PJE et al., The epidemiology of wound infection. A 10- year prospective study of 62,939 wounds. Surg. Clin. North Am. 1980; 60(1):27-40.

Josep Ballus et al., Surgical site infection in critically ill patients with secondary and tertiary peritonitis: epidemiology, microbiology and influence in outcomes. BMC Infect Dis. 2015; 15(304):1-6.

Lilani, S.P., et al., Surgical site infections in clean and clean - contaminated cases. IJMM. 2005; 23(4):249-52.

Mandakini Pawar et al., Resistance in gram negative bacilli in a Cardiac Intensive care unit in India: Risk factors and outcome. Ann Card Anaesth. 2008; 11(1): 20-6. Available from: http://www.annals.in/text.asp?2008/11/ $1 / 20 / 38445$.

Martone et al., Recognition, prevention, surveillance, and management of surgical site infections. Clin Infect Dis. 2001; 33: S67-S106.

Mohanty S., et al., Bacteriological and antimicrobial susceptibility profile of soft tissue infections from Northern India Year. IJMS. 2004; 58(1):10-15. Available at http://hdl.handle.net/1807/2487.

Nichols RL. Prevention of infection in high risk gastrointestinal surgery. Am. J. Med. 1984; 76: 111-9.

Rao AS et al., Postoperative wound infections. J Indian Med. Assoc. 1975; 64: 90-3.

Ronald Lee Nichols. Preventing surgical site infections: A surgeon's perspective. EID. 2001; $\quad 7(2)$. DOI: 10.3201/eid0702.700220

Singh G et al., Necrotising infections of soft tissues - a clinical profile. Eur J Surg. 2002; 168:366-71.

Suchitra Joyce et al., Costs Associated With Infection Control Strategies In Aresource Constrained Setting. Afr. J. Microbiol. Res. 2009; 3(4): 175-79.

\section{How to cite this article:}

Sagila Savithri Gangadharan. 2018. A Study on Post-Operative Wound Infections. Int.J.Curr.Microbiol.App.Sci. 7(02): 1504-1511. doi: https://doi.org/10.20546/ijcmas.2018.702.181 